\title{
Direct analysis of microbial populations in soil and fresh- water aquifers using nucleic acid based techniques
}

\author{
Carsten S. Jacobsen, Julia R. de Lipthay, Mikkel Bender, Line Fredslund, Anders R. Johnsen and \\ Kaare Johnsen
}

The first DNA-based methods for direct quantification of soil protozoa, and a DNA-based quantification method to describe the spread of phenanthrene-degrading bacteria in soil and freshwater aquifers, have recently been developed at the BIOPRO Research Centre at the Geological Survey of Denmark and Greenland (GEUS). Well-known genes for phenoxyalcanoic acid degradation have been used to monitor the in situ degradation of phenoxyalcanoic acid pesticides. Studies have been initiated on the short-lived mRNA molecules that are expected to provide a shortcut to the understanding of low, yet important, microbial activity in geological samples. This article reviews recent developments

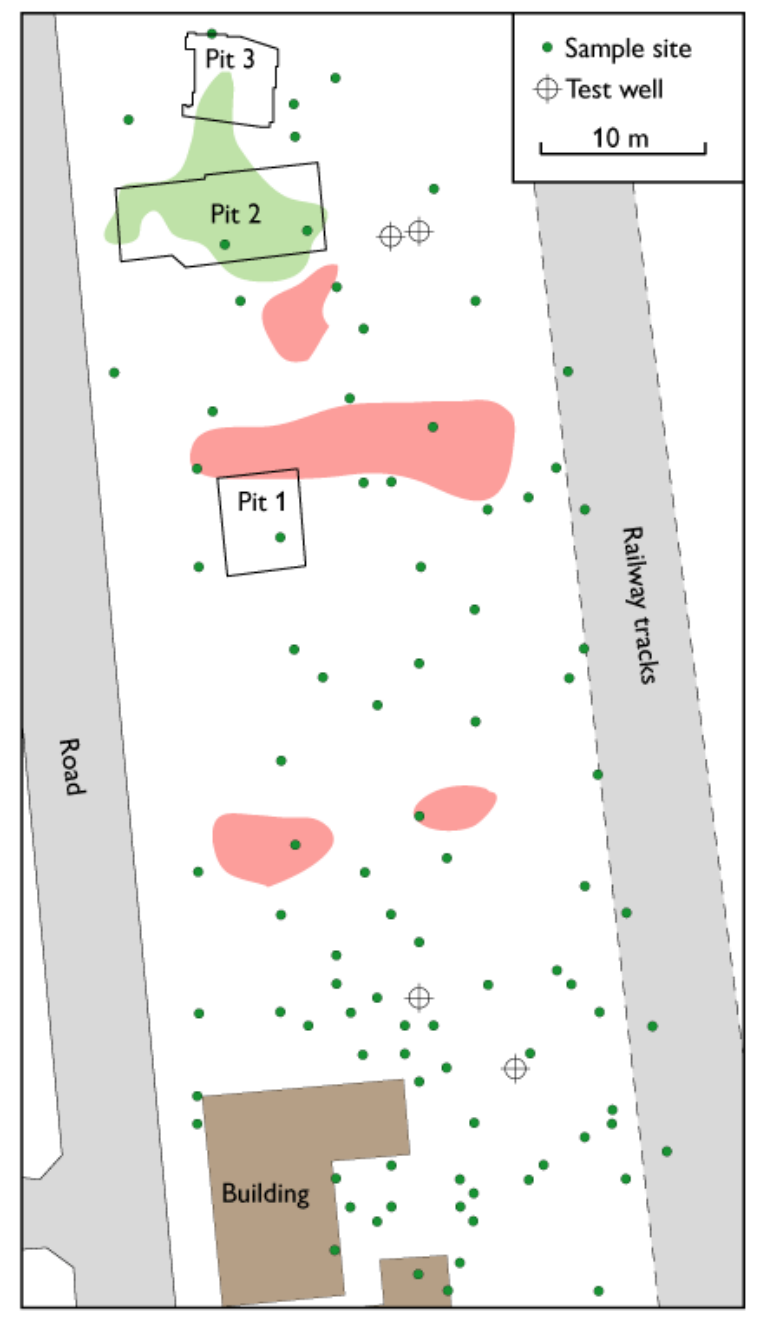

in techniques based on analysis of nucleic acids from soils and aquifers.

Analytical work has been carried out mainly on soil samples from a former asphalt production plant at Ringe (Fig. 1). The Ringe plant constitutes one of the most polluted industrial sites in Denmark, and is a priority site of studies by the BIOPRO Research Centre. Although rich in carbon, the Ringe subsoil is an oligotrophic environment due to the high content of polycyclic aromatic hydrocarbons (PAH). This is an environment where the supply of nutrients to microorganisms is low, leading to slow growth, low total numbers of microorganisms and small cells. To study microbial communities of oligotrophic environments, analytical methods with low detection limits are needed. Until recently, microorganisms of natural environments were mainly studied by cultivation-dependent methods. However, microorganisms that can be cultured on agar plates are now known to represent only a small fraction of the total microbial community. Modern methods, therefore, need to be based on the detection of biomolecules in the microorganisms rather than being dependent on growth of the microorganisms. The best available techniques are based on DNA and RNA molecules (Fig. 2), which due to their high level of resolution allow closely related organisms or functional genes to be distinguished. In the following review, examples are given of applications of these nucleic acid based methods.
Fig. 1. Location of the former asphalt plant at Ringe, one of the most polluted industrial sites in Denmark. The BIOPRO Research Centre at GEUS monitored in situ degradation of phenoxyalcanoic acid pesticides. The pits, sampling sites and wells were used to determine the distribution of contamination of tar (pale red) and kerosene (green) in the subsoil. A field injection experiment, using a mixture of six different herbicides was carried out at a shallow sandy aquifer at Vejen. Modified from Rosenbom et al. (2000). 


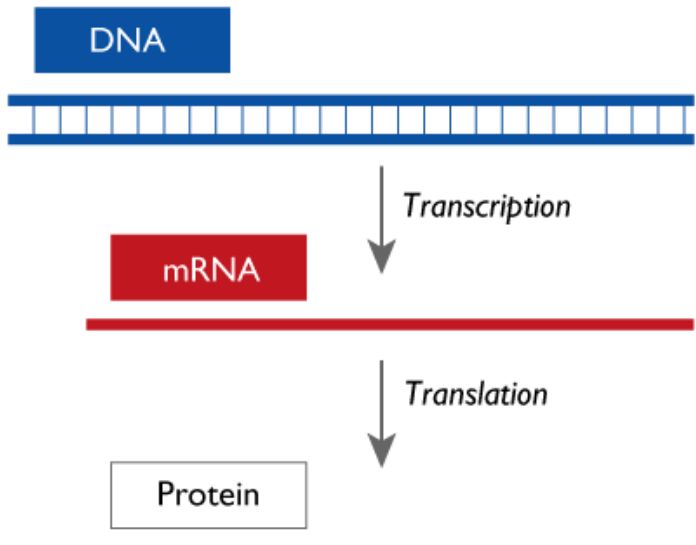

Fig. 2. All information regarding cell function and structure is contained as a genetic code in the cell DNA. Each gene encodes a specific function by dictating the synthesis of a specific protein. Before proteins are synthesised, the genes are copied (transcribed) into messenger RNA (mRNA). After transcription, the mRNA is translated into protein by protein-synthesising machinery called ribosomes. The sequence of bases in the mRNA, copied after the base sequence in the gene, determines the structure and function of the protein.

\section{DNA analysis is not limited to living organisms}

Unlike other microbial analysis tools, DNA analyses may be carried out on both living and dead material, as long as the DNA has not been degraded. For analysis of DNA in soils, a major problem is the presence of humic substances, that even at low concentrations interfere with the enzymes used in the DNA amplification process. All research groups working with soil nucleic acids experience problems with amplification of DNA when soils contain much humic material. One way to solve this problem is to use selective purification methods in which single-stranded DNA is selectively purified from the soil using a molecular 'fishing rod' equipped with the complementary DNA strand.

After extracting the gene of interest, it is possible to multiply its numbers using the 'Polymerase Chain Reaction' (PCR; Saiki et al. 1985). PCR is an exponential reaction in which a single DNA strand can, in principle, produce four million identical copies by 25 cycles of multiplication. In PCR, two small DNA sequences, corresponding to two regions on the gene, are selected as priming sites for two segments of complementary DNA. These small DNA pieces are designated 'primers'. The primers serve as the target of the PCR, and lead to the formation of a large number of DNA molecules identical to the original gene. The primers are constructed by consulting DNA sequence databases on the Internet. Since these databases are very comprehensive, it is possible to construct primer sets that are specific for the desired taxonomic or functional groups.
This technique has, for example, enabled forensic experts to produce enough DNA molecules to determine whether the genomic fingerprint of a person matches that of a bloodstain at the scene of a crime. We have used this technique widely for the analysis of microorganisms in the environment, and the detection limit is less than 40 cells in a sample (Jacobsen 1995). The challenge is now not only to detect, but also to quantify the DNA from very few cells in soil and freshwater aquifers.

\section{Quantitative DNA techniques for the enumeration of soil flagellates and bacteria}

As a consequence of the exponential nature of PCR, it is an excellent technique for the detection of specific DNA sequences. On the other hand, it is not quantitative, and small differences in the efficiency of the reaction affect the final number of DNA copies. Several methods have been proposed to resolve this problem. One possibility is using the principle of 'most probable number' (MPN) estimates, where the DNA template is serially diluted in several replicate reactions. By looking at which dilutions of template DNA that give a product from the PCR, and in how many of the replicate reactions, an estimate of the original number of genes in the sample can be made. This approach has been used to develop the first successful molecular detection and quantification of protozoa in soil (Fredslund et al. 2001). This new technique represents a breakthrough in reliable enumeration of soil protozoa, since these often small and amoeboid organisms are difficult to enumerate using microscopy techniques. Traditionally, the enumeration was based on growth-dependent techniques, where the cultivable fraction of the total protozoan populations was not known. In our study, a part of the $18 \mathrm{~S}$ rDNA of the common soil flagellate Heteromita globosa was sequenced and PCR primers for this gene were developed. In a sterilised soil at the Ringe asphalt production plant, the population dynamics of this flagellate and the phenanthrene-degrading bacterium Pseudomonas putida OUS82 were quantified using both growth-dependent techniques and the MPN-PCR assay (Fredslund et al. 2001).

Alternative methods for quantification of DNA are the real-time PCR and the competitive PCR methods. In realtime PCR, the DNA multiplication is monitored on-line using a combination of fluorescent DNA stains and fibre optics coupled to a computer. GEUS has recently received funding from the Danish Natural Science Research Council to implement this technique.

Competitive PCR makes use of an internal standard in the form of a similar, but shorter DNA molecule, which is recognised and hence amplified by the same primer set as the template DNA (the DNA that needs to be quantified). The 


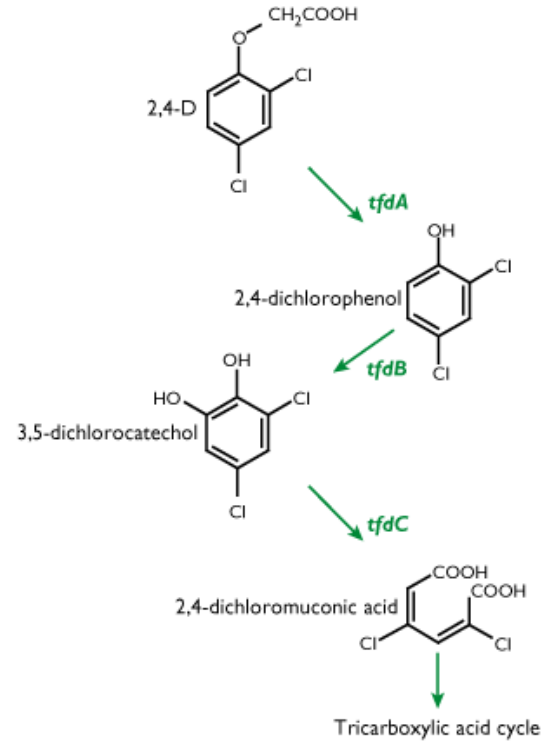

Fig. 3. Pathway for degradation of 2,4-D as elucidated in the bacterial strain Ralstonia eutropha JMP134. The $t f d A$ gene encodes a 2,4-D dioxygenase, $t f d B$ encodes a 2,4-dichlorophenol hydroxylase, and $t f d C$ encodes a chlorocatechol 1,2-dioxygenase. The 2,4-dichloromuconic acid generated by the activity of the $t f d C$ gene product is further transformed to intermediates of the tricarboxylic acid cycle by the activity of other $t f d$ gene products.

internal standard is added to the reaction mixtures in decreasing amounts and competes with the template DNA for amplification. Thus, the amount of product from the internal standard is inversely related to the initial amount of the template DNA. Competitive PCR exploits the highly sensitive nature of the PCR process, while using an internal standard to bypass the quantification problems inherent in the amplification reaction (Johnsen et al. 1999).

\section{Microbial changes in aquifers contaminated with phenoxyalcanoic acid herbicides}

This section focuses on specific genes of interest rather than on organisms. Phenoxyalcanoic acid herbicides are extensively used in agriculture, and include compounds such as mecoprop (MCPP), 2,4-dichlorophenoxyacetic acid (2,4-D) and dichlorprop. A common pathway for 2,4-D degradation has been determined for the bacterial strain Ralstonia eutropha JMP134, and the catabolic genes ( $t f d)$ encoding the specific enzymes have been identified (Fig. 3; Don et al. 1985). The in situ adaptation processes of the indigenous microorganisms when exposed to these herbicides have been investigated by studying the impact on a microbial community in a freshwater aquifer. A field injection experiment was carried out at a shallow sandy aquifer at Vejen, Denmark (Fig. 1). During a seven-month period, a mixture of six different herbicides, including MCPP and dichlorprop, was continuously injected into the aquifer creating a contaminant plume (Broholm et al. 2000).

Sediment and groundwater samples from herbicideexposed ( 1 and 2 ) and non-exposed (NX) sites (Fig. 4A) were collected, and the impact on microbial community structure and function was studied (de Lipthay et al. 2000). Laboratory incubations demonstrated that sediment samples collected inside the contaminant plume had acquired a significantly increased capacity for herbicide mineralisation compared to samples from non-exposed sites (Fig. 4A). Thus, the in situ exposure to herbicides resulted in microbial communities that were better adapted to the degradation of phenoxyalcanoic acids. This was further demonstrated by greatly increased populations of pesticide degraders inside the pesticide plume, both when enumerated by cultivation, and when quantified by the number of pesticide genes ( $f d A B C$ ) detected by PCR methods (Fig. 4B, treatment 1 and 2). Pesticide degraders and their $t f d$ genes were undetectable outside the plume (Fig. 4B, treatment NX). The most likely

A

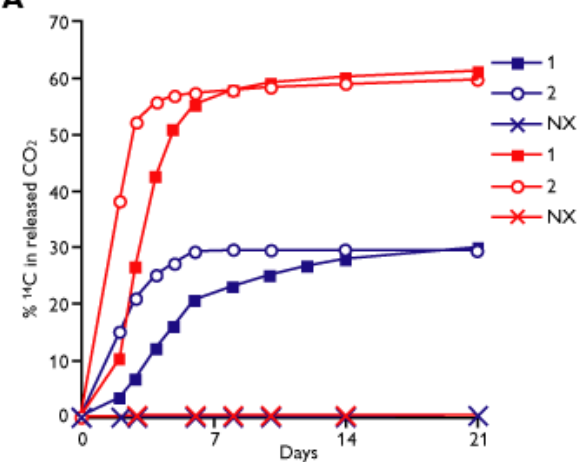

B

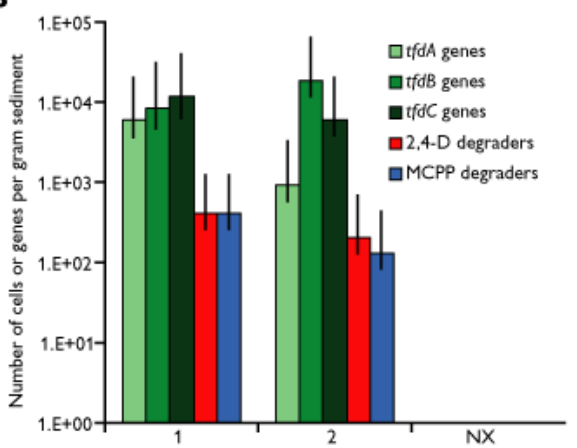

Fig. 4. A: Mineralisation of the phenoxyalcanoic acid herbicides 2,4-D (red) and MCPP (blue) in laboratory incubations of sediment samples from herbicide exposed $(1,2)$ and non-exposed (NX) sites of the Vejen aquifer. B: Effect of $i$ situ herbicide exposure in two exposed $(\mathbf{1}, \mathbf{2})$ sites on microbial biomass of 2,4-D and MCPP degraders, and on the presence of $t f d A, t f d B$ and $t f d C$ genes. Data show that indigenous microbial communities carry the $t f d$ genes and are capable of degrading phenoxyalcanoic acids 
explanation is that microorganisms carrying the $t f d$ genes had a selective advantage in that they could make use of the pesticides as sources of carbon and energy. The data suggest that natural attenuation is a likely procedure for clean up of this group of herbicide compounds when originating from pointsource contaminations.

\section{Analysis of microbial activity applying mRNA techniques}

The presence or absence of specific microorganisms may be determined by use of cultivation or DNA-based techniques, although these methods give no information as to whether the organisms are actually active in the environment. Microbial activity may be measured in several ways. The first sign of activity in microbial cells is the synthesis of messenger-RNA (mRNA; Fig. 2). These molecules have half-lives of only a few minutes, and the detection of mRNA thus ensures that the genes of interest are actually expressed at the time of sampling. Another approach is to detect the activity of the gene products - the enzymes. However, the longevity of enzyme activities is variable. A third approach is to measure the target molecules of the enzymes, i.e. the pollutant molecules. By use of analytical chemical methods such as gas and liquid chromatography, the concentration of target molecules may be measured, and the dissipation of pollutants indicates microbial activity.

A major topic of future studies in microbial ecology will be the assessment of microbial activity by the application of mRNA techniques to answer which microorganisms are active, and under which conditions their genes are expressed.

The two most commonly used techniques for detection of mRNA (Fig. 2) are reverse transcription polymerase chain reaction (RT-PCR) and RNA-RNA hybridisation.

In RT-PCR, the first step is a reverse transcription process the conversion of mRNA into copy DNA (cDNA). Reverse transcription requires a small DNA primer to bind to the mRNA in order to initiate synthesis of cDNA. Thus, specific mRNAs can be amplified by using sequence-specific primers in the RT-PCR reaction. Following synthesis of cDNA, a normal PCR is carried out to multiply the cDNA, and the resulting PCR products are detected by usual DNA detection techniques.

Direct RNA-RNA hybridisation analyses exploit a completely different principle; this is directly quantitative but lacks the sensitivity of RT-PCR. First, the total content of mRNA is extracted. Then the mRNA of interest is identified by binding of a specific RNA probe with a sequence comple- mentary to the mRNA gene of interest (hybridisation). By using a 'radiolabelled' probe, the final quantification of the mRNA of interest is easily done by determining the amount of 'radiolabelled' bound to the mRNA.

A study using freshwater samples artificially contaminated with the herbicide 2,4-D, revealed a significant increase in the amount of $t f d A$ mRNA, as measured by hybridisation of RNA extracts using a $t f d A$ specific probe. This demonstrates that the 2,4-D degraders in the freshwater samples were actively degrading the 2,4-D (Fig. 5). Transcription of tfdA was, however, transient and the degradation of 2,4-D continued although mRNA levels dropped. These observations illustrate that the herbicide-degrading enzymes encoded by the mRNA last longer in the cells than the mRNA 'signal' itself.

\section{References}

Broholm, M.M., Rügge, K., Tuxen, N., Mosbæk, H., \& Bjerg, P.L. 2000: Migration and degradation of pesticides in an aerobic groundwater aquifer: Field injection experiments. In: Bjerg, P.L., Engesgaard, P. \& Krom, T.D. (eds): Proceedings of the International Conference on Groundwater Research, Copenhagen, 169-170. Rotterdam: Balkema.

de Lipthay, J.R., Johnsen, K., Aamand, J., Tuxen, N., Albrechtsen, H.-J. \& Bjerg, P.L. 2000: Continuous exposure of pesticides in an aquifer changes microbial biomass, diversity and degradation potential. In: Bjerg, P.L., Engesgaard, P. \& Krom, T.D. (eds): Proceedings of the International Conference on Groundwater Research, Copenhagen, 157-158. Rotterdam: Balkema.

Don, R.H., Weightman, A.J., Knackmuss, H.-J. \& Timmis, K.N. 1985: Transposon mutagenesis and cloning analysis of the pathway for degradation of 2,4-dichlorophenoxyacetic acid and 3-chlorobenzoate in Alcaligenes eutrophus JMP134(pJP4). Journal of Bacteriology 161(1), 85-90.

Fredslund, L., Ekelund, F., Jacobsen, C.S. \& Johnsen, K. 2001: Development and application of a Most Probable Number-PCR assay to quantify flagellate populations in soil samples. Applied and Environmental Microbiology 67(4), 1613-1618.

Jacobsen, C.S. 1995: Rapid microscale detection of specific bacterial DNA in soil using magnetic capture-hybridization and polymerase chain reaction amplification assay (MCH-PCR). Applied and Environmental Microbiology 61(9), 3347-3352.

Johnsen, K., Enger, Ø., Jacobsen, C.S., Thirup, L. \& Torsvik, V. 1999: Quantitative selective PCR of 16 S ribosomal DNA correlates well with selective agar plating in describing population dynamics of indigenous Pseudomonas spp. in soil hot spots. Applied and Environmental Microbiology 65(5), 1786-1789.

Rosenbom, A.E., Klint, K.E.S., Fredericia, J., Springer, N. \& Andersen, G. 2000: Pore-to-core scale-up studies of the transport properties of organic pollutants with natural attenuation. Danmarks og Grønlands Geologiske Undersøgelse Rapport 2000/79, 39 pp.

Saiki, R.K., Scharf, S., Faloona, F., Mullis, K.B., Horn, G.T., Erlich, H.A. \& Arnheim, N. 1985: Enzymatic amplification of B-globin genomic sequences and restriction site analysis for diagnosis of sickle cell anemia. Science 230, 1350-1354.

\section{Authors' address}

Geological Survey of Denmark and Greenland, Øster Voldgade 10, DK-1350 Copenhagen K, Denmark. E-mail: csj@geus.dk 